\title{
Extremely rare case of a rupture of the left sinus of Valsalva aneurysm into the main pulmonary artery
}

\author{
Virendra C. Patil, Harsha V. Patil, Sanjay Kshirsagar, Bhupal Pujari \\ Department of Medicine, Krishna Institute of Medical Sciences University, Karad, \\ Satara - 415 110, Maharashtra, India \\ Address for correspondence: Dr. Virendra C. Patil, Department of Medicine, Krishna Institute of Medical \\ Sciences University, Dhebewadi Road, Karad, Satara - 415 110, Maharashtra, India. \\ E-mail:virendracpkimsu@rediffmail.com
}

\begin{abstract}
We describe a case of ruptured aneurysm of the left coronary sinus of Valsalva diagnosed by transthoracic two-dimensional echocardiography. A very few cases with ruptured left sinus of Valsalva have been reported. We are reporting a rupture of the left sinus of Valsalva into the main pulmonary artery in a 40-year-old male diagnosed by transthoracic echocardiography.
\end{abstract}

Key words: Rupture into the main pulmonary artery, sinus of Valsalva, transthoracic echocardiography

\section{INTRODUCTION}

Sinus of Valsalva aneurysm is a rare congenital defect and the diagnosis is usually made after it ruptures. The anomaly most often involves the right coronary sinus and less frequently the noncoronary sinus. Since the aortic valve occupies a central position in the base of the heart, a rupture of a sinus of Valsalva aneurysm can occur in any of the four heart chambers. The rupture of the left sinus of Valsalva into the pulmonary artery is extremely rare. ${ }^{[1,2]}$

\section{CASE REPORT}

A 40-year-old patient presented with acute onset dyspnea, ascites, and peripheral edema. Physical examination revealed a continuous murmur (Grade 4/6) across the

\begin{tabular}{|l|l|}
\hline \multicolumn{3}{|c|}{ Access this article online } \\
\hline Quick Response Code: & Website: \\
\hline & www.jcdronline.com \\
\cline { 2 - 3 } & \\
\hline
\end{tabular}

precordium. Blood pressure was 160/60 $\mathrm{mmHg}$ with bilateral basal rales. Jugular venous pressure was raised.

\section{INVESTIGATIONS}

Hemoglobin was $12 \mathrm{gm} \%$. The total count was 8700 with normal differentiation. Renal and liver functions were normal. A chest X-ray showed an enlarged cardiac shadow and pulmonary congestion. The electrocardiogram revealed right ventricular hypertrophy with a prolonged PR interval (first AV block).

Transthoracic two-dimensional echocardiography (TTE) showed a normal left ventricular systolic function. The overall left ventricular ejection fraction was $70 \%$. The left ventricular wall thickness and function was normal. There was severe aortic regurgitation. All cardiac chambers were dilated with moderate pulmonary, mitral, and tricuspid regurgitation with severe pulmonary hypertension (pulmonary artery pressure: $42.9 \mathrm{mmHg}$ ). At the aortic root level, there was an aortopulmonary communication indicating a rupture of the left sinus of Valsalva. The pulmonary artery was dilated. The color Doppler images showed the blood shunting from the aorta to the pulmonary artery. The parasternal short-axis view with the 
Patil et al:: A rupture of the left sinus of Valsalva aneurysm into the main pulmonary artery

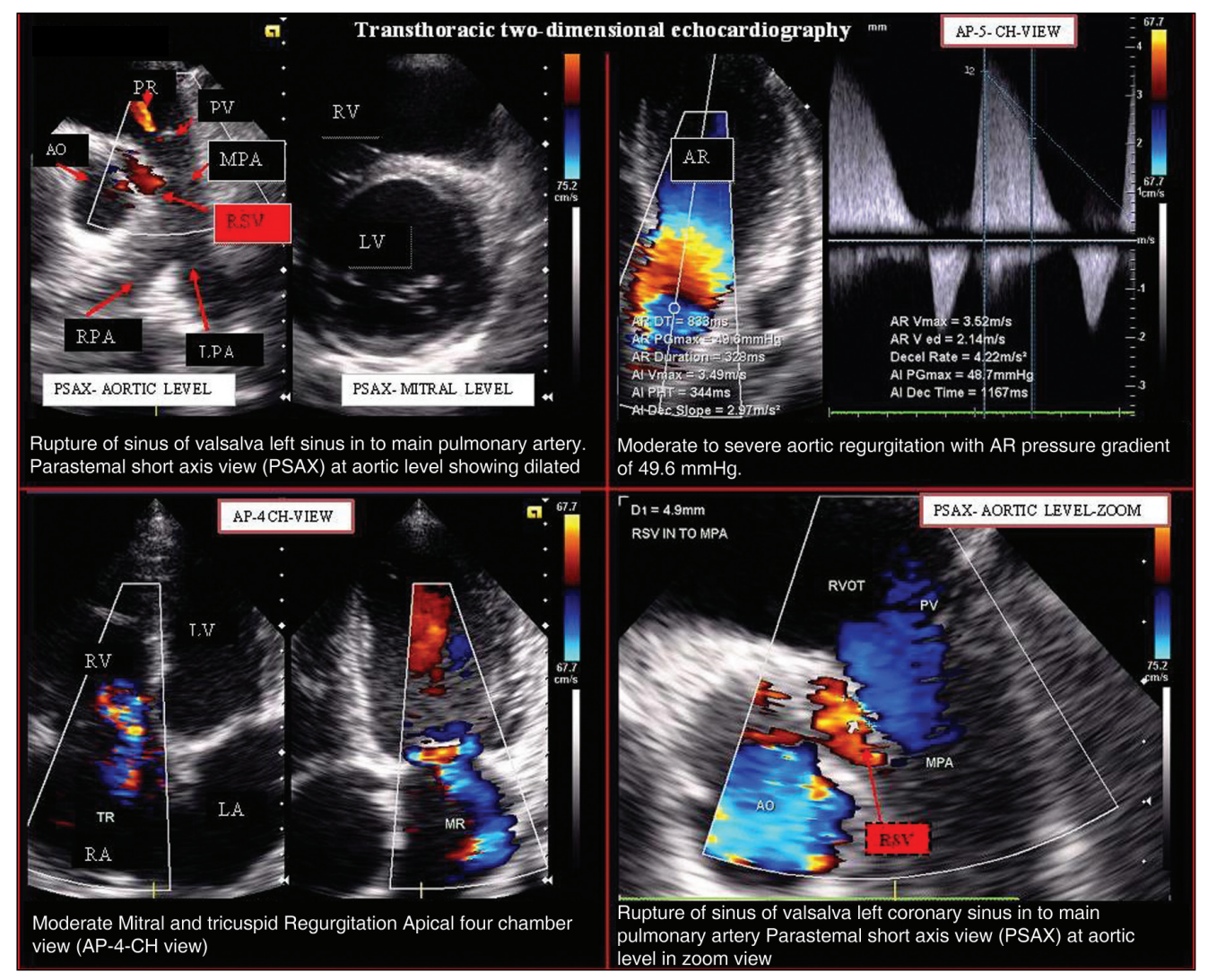

Figure 1: Transthoracic two-dimensional echocardiography, shows the rupture of the left sinus of Valsalva, into the main pulmonary artery, severe aortic regurgitation, moderate mitral regurgitation, moderate tricuspid regurgitation, moderate pulmonary regurgitation and all dilated cardiac chambers

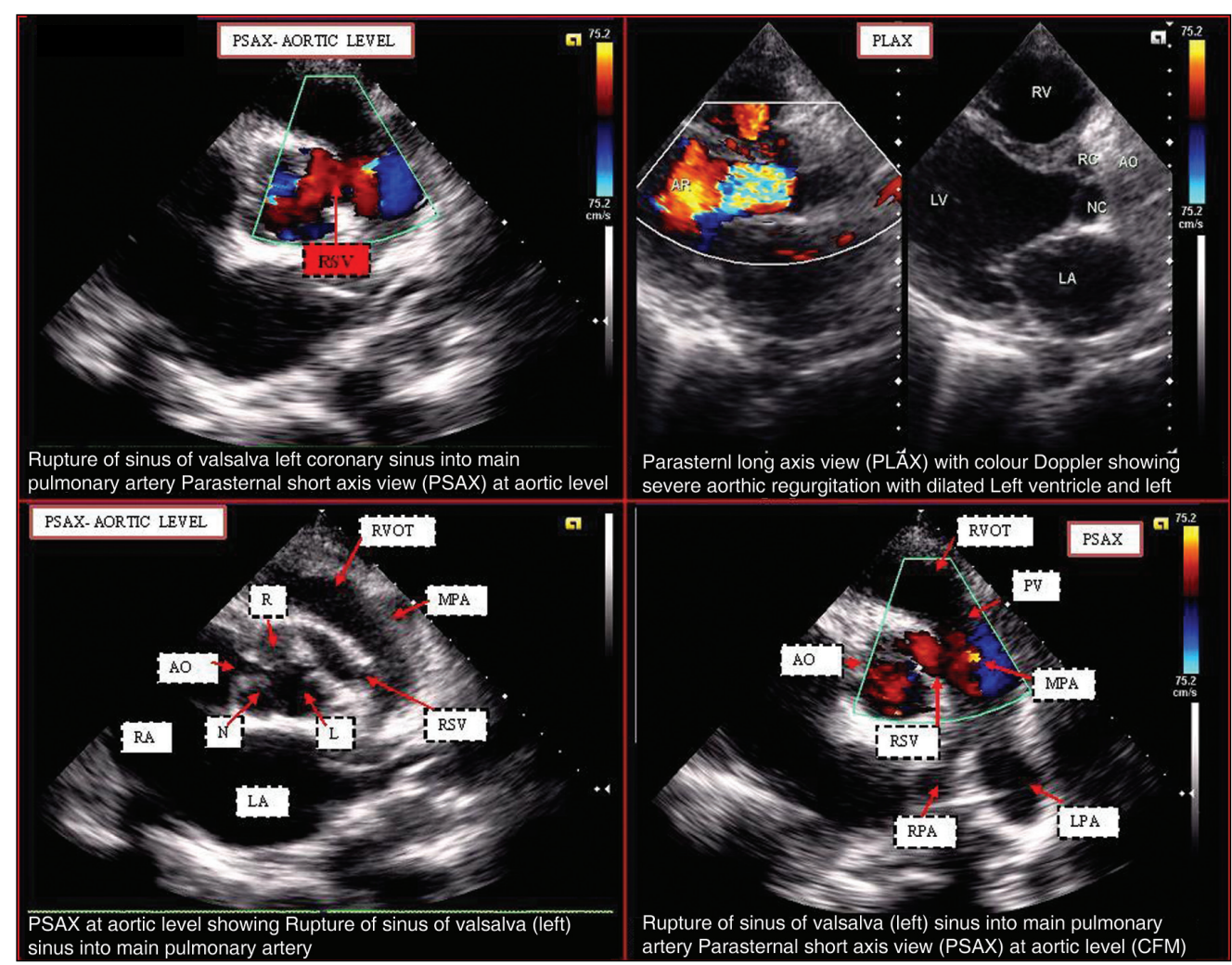

Figure 2: Transthoracic two-dimensional echocardiogram showing the rupture of the left coronary sinus of Valsalva into the main pulmonary artery, and severe aortic regurgitation 
color Doppler technique showed the shunting blood flow from the left sinus of Valsalva through the "wind sock" into the main pulmonary artery [Figures 1 and 2].

The patient was treated for congestive cardiac failure and referred to a cardiologist and cardiothoracic surgeon for further evaluation and repair. Unfortunately, in the cardiac center the, patient succumbed to death after an episode of ventricular tachycardia and congestive cardiac failure.

\section{DISCUSSION}

Previously, the diagnosis of a ruptured sinus of Valsalva aneurysm in a living patient was rare, with most of the reports coming from autopsy or surgery. ${ }^{[2]}$

Nowadays, the diagnosis is possible with both TTE and transoesophageal echocardiography (TEE). In the patient presented, TTE provided very detailed information to the surgeon. We obviously would have performed a TEE had we not reached a precise anatomical description with TTE. ${ }^{[2,3]}$ Aneurysms of the sinus of Valsalva account for less than $1 \%$ of congenital cardiac anomalies. ${ }^{[4]}$

A total of $90-95 \%$ of these congenital aneurysms originate in the right or noncoronary sinus and project into the right ventricle or into the right atrium. Almost all aneurysms arising in the noncoronary sinus rupture into the right atrium and those arising in the right coronary sinus generally communicate with the right ventricle and occasionally with the right atrium. ${ }^{[5,6]}$

In the present case report, a patient showing features of congestive cardiac failure with continuous murmur with acute aortic regurgitation and wide pulse pressure was diagnosed by TTE. Fazio et al..$^{[2]}$ reported a similar and rare case of a rupture of the left sinus of Valsalva aneurysm into the pulmonary artery. Complications of the sinus of Valsalva aneurysms include aortic regurgitation, coronary artery flow compromise, arrhythmias, and rupture. Most commonly, the rupture occurs from the right coronary sinus into the right ventricle or into the right atrium. However, the rupture may also occur into the pericardium, the pleural space, or the left heart chambers. ${ }^{[6]}$

\section{CONCLUSION}

A clinician should always remember the unusual etiology of a common aortic valvular lesion like acute aortic regurgitation; in this case report, the etiology is a rupture of the left sinus of Valsalva aneurysm into the pulmonary artery. In the present case, anatomical imaging was achieved enough by TTE to diagnose the rupture of the left sinus of Valsalva aneurysm into the pulmonary artery.

\section{REFERENCES}

1. Kar AK, Bhattacharya S, Ray D, Mondal M, Ghosh S, Mazumdar A. Rupture of the sinus of Valsalva into the pulmonary artery. Indian Heart J 2002;54:415-7.

2. Fazio G, Zito R, Dioco DD, Mussagy C, Loredana S, Damasceno A, et al. Rupture of a left sinus of Valsalva aneurysm into the pulmonary artery. Eur J Echocardiogr 2006;7:230-2.

3. Iadanza A, Fineschi M, Del Pasqua A, Pierli C. Echocardiography diagnosis of ruptured congenital right coronary sinus of Valsalva aneurysm into right ventricle. Eur J Echocardiogr 2006;7:387-9.

4. Dev V, Goswami KC, Shrivastava S, Bahl VK, Saxena A. Echocardiographic diagnosis of aneurysm of the sinus of Valsalva. Am Heart J 1993;126:930-6.

5. Rothbart RM, Chahine RA. Left sinus of Valsalva aneurysm with rupture into the left ventricular outflow tract: diagnosis by color-encoded Doppler imaging. Am Heart J 1990;120: 224-7.

6. Rigo T, Zeppellini R, Cucchini F. Rupture of an aneurysm of the noncoronary sinus of Valsalva into the right atrium: The "wind sock" echocardiographic appearance. Ital Heart J 2001;2:237-8.

Source of Support: Nil, Conflict of Interest: None declared. 